\title{
GIGAHERTZ BANDWIDTHS IN MULTIMODE, LIQUID-CORE, OPTICAL FIBRE WAVEGUIDE
}

\author{
W.A. GAMBLING, D.N. PAYNE and H. MATSUMURA* \\ Department of Electronics, University of Southampton, Southampton, UK
}

Received 6 October 1972

\begin{abstract}
An attenuation of $7 \mathrm{~dB} / \mathrm{km}$ has been achieved over kilometre lengths of liquid-core, multi-mode op tical fibre waveguide. The measured pulse dispersion depends on the mode distribution launched and on mode conversion which is a function of bend radius. Using a laser source a dispersion corresponding to a bandwidth $\approx \mathrm{l} \mathrm{GHz}$ has been achieved and does not appear to be limited by fibre imperfections.
\end{abstract}

\section{Introduction}

Single-mode optical fibres are expected to exhibit larger bandwidths than multimode fibres since, in the latter, mode conversion, which can arise from bends or inhomogeneities, is expected to cause an increased dispersion. In single-mode fibres such mode conversion effects give rise to increased attenuation rather than increased dispersion. On the other hand single-mode fibres are more difficult to manufacture, require great precision in both launching and jointing, and the attenuation is critically dependent on both core and cladding materials. In multimode fibres these technological difficulties are greatly reduced and the cladding material has only a second-order effect on the overall fibre transmission loss.

If the light propagating in multimode fibres scatters into all possible modes then the bandwidth is only a few megahertz. However, measurements in glass-core multimode fibres of pulse dispersion [1] have shown that bandwidths in the region of $100 \mathrm{MHz}$ may be possible under optimum launching conditions. As a result of these measurements and the achievement of attenuations in liquid-core [2] and silica [3] fibres of less than $10 \mathrm{~dB} / \mathrm{km}$, as well as the ease of handling, multimode fibres have now become of greatly increased interest.

* On leave from the Nippon Sheet Glass Co.
A recent analysis [4] using a propagation model based on rays reflecting specularly at the core/cladding interface and assuming no mode conversion gives results in reasonable agreement with experiment but the experimental dispersion is somewhat greater than that predicted by the theory. This indicates that some mode conversion may be occurring and typical causes of this could be scattering in the core or at the core/cladding interface, or the effect of curvature of the fibre. We have investigated the latter effect with glass-core fibres by rewinding samples onto drums of different diameter and the measurements show that the dispersion decreases as the bend radius is increased. As part of our study of liquid-core fibres we have repeated the measurements and obtain a clear correlation between curvature of the fibre axis and mode conversion, through measurements of propagation delay, pulse dispersion and angular distribution of light in the core.

\section{Experiment}

The fibres used [2] in the measurements consisted of a capillary of ME 1 glass of $50 \mu \mathrm{m}$ in ternal diameter filled with hexachlorobuta-1,3-diene. The attenuation was measured over a length of $1 \mathrm{~km}$ at a wavelength of $1.08 \mu \mathrm{m}$ and is $7.3 \mathrm{~dB} / \mathrm{km}$ although measurements made at an independent laboratory [5] give a value of $6 \mathrm{~dB} / \mathrm{km}$. The at tenuation is similarly low [2] over the range 1.04 
to $1.10 \mu \mathrm{m}$. Most of the results to be described were obtained with a length of $200 \mathrm{~m}$ but confirmatory checks were also made over $400 \mathrm{~m}$. The numerical aperture of the fibre is 0.46 .

As described elsewhere [4] 0.6 nsec pulses at an $80 \mathrm{MHz}$ repetition rate were produced by a modelocked $\mathrm{TEM}_{00}$ helium/neon laser and were transmitted along the fibre. The pulse dispersions quoted refer to the difference between the half-power pulse durations of the input and output pulses as monitored by EMI S30500 avalanche photodiodes. The specific dispersions shown in figs. $1 \mathrm{a}$ and $2 \mathrm{a}$ are obtained by averaging over the length of fibre used. We thus define "pulse dispersion" as the measured increase in pulse half-width and "specific dispersion" as the average pulse broadening per unit length. Measurements over a range of lengths indicate that the specific dispersion is almost constant along the fibre, maximum departure being in the fibre section immediately following the launch.

The angular width of the input beam was varied by using lenses of varying focal lengths and the beam waist of the gaussian laser beam was positioned centrally at the entry face of the fibre in the supporting mount. The output beam divergence was measured by scanning a PIN photodiode over the radiation pattern emerging from the output mount. The angular widths shown in terms of the beam semiangles in figs. 1 and 2 are those where the beam intensity has fallen to $e^{-2}$ times that on the axis. For the measurements at different bend radii the fibres were rewound onto expanded polystyrene drums of diame ters $11,15,21,40,60,91$ and $194 \mathrm{~cm}$. For the large diameters it was found to be important to ensure that the drum circumference was smooth.

\section{Results}

The curves of fig. 1a show that for a given angular width of the input beam the pulse dispersion falls with increasing bend radius. As predicted by the ray propagation model [4], and as already shown for glass-core fibres, the dispersion is greater at large angular beam widths. The figure also shows that with a $0.3^{\circ}$ beam the dispersion is only $1.6 \mathrm{psec} / \mathrm{m}$ at a bend radius of $1 \mathrm{~m}$ and hence the maximum permissible pulse rate is nearly $1 \mathrm{Gbit} / \mathrm{sec}$. The curve falls steeply at large bend radii and it will be interesting to see whether a much larger pulse rate is possible in a straight fibre and what the limitation due to fibre scattering and imperfections will be. The steep slope of this lower curve of dispersion as a function of bend radius explains the sensitivity of the measurement, and its repeatability, to the smoothness of the drum circumference. The results suggest that the higher dispersion found with increased curvature of the fibre arises from mode conversion. The pulse rate which can be achieved in a straight line under appropriate launching conditions should therefore exceed $1 \mathrm{Gbit} / \mathrm{sec} \mathrm{km}$ and will ultimately be limited by fibre inhomogeneities, although no limitation due to the latter has yet been ubserved.

The fact that a redistribution of light in the fibre is occurring is shown by the increase in output beam divergence with decreasing bend radius. Fig. I b clearly indicates that the fibre output beam divergence is greater than that injected at the input end, the difference being most evident for small bend radius. Further investigations of the effect reveal that the angular distribution of light increases rapidly from the initial value over the first few tens of metres, and thereafter remains relatively constant at the values given in fig. $1 \mathrm{~b}$, which are (i) a function only of the input angular width and the bend radius of the fibre, (ii) appreciably less than the acceptance angle of the fibre.

The curves of fig. $1 \mathrm{~b}$ are remarkably similar in shape to those of dispersion in fig. 1 a, although the relationship between angular width and pulse dispersion is not a linear one [4]. Measurements of pulse dispersion with increasing fibre length show an approximately linear relationship, i.e., the specific dispersion ( $\mathrm{psec} / \mathrm{m}$ ), which is the quantity related to angular width, is almost independent of length. It must be noted however that the accuracy of our dispersion measurements $(\approx 0.1$ nsec) is not yet high enough at short lengths where the angular width rapidly increases from the value at launch and the dispersion/unit length is expected to depart from the constant value. Nevertheless because the length over which the specific dispersion is expected to depart from the constant value is relatively small then the error incurred by averaging the cibserved dispersion over the fibre leng th to obtain the specific dispersion is negligible. Thus for the range of lengths in the experiments described here $(200-400 \mathrm{~m})$ both the specific dispersion and the output beam width are largely independent of fibre length.

Figs. 1 a and $1 b$ show clearly the effect of bend radius on dispersion and angular distribution of light in the core 

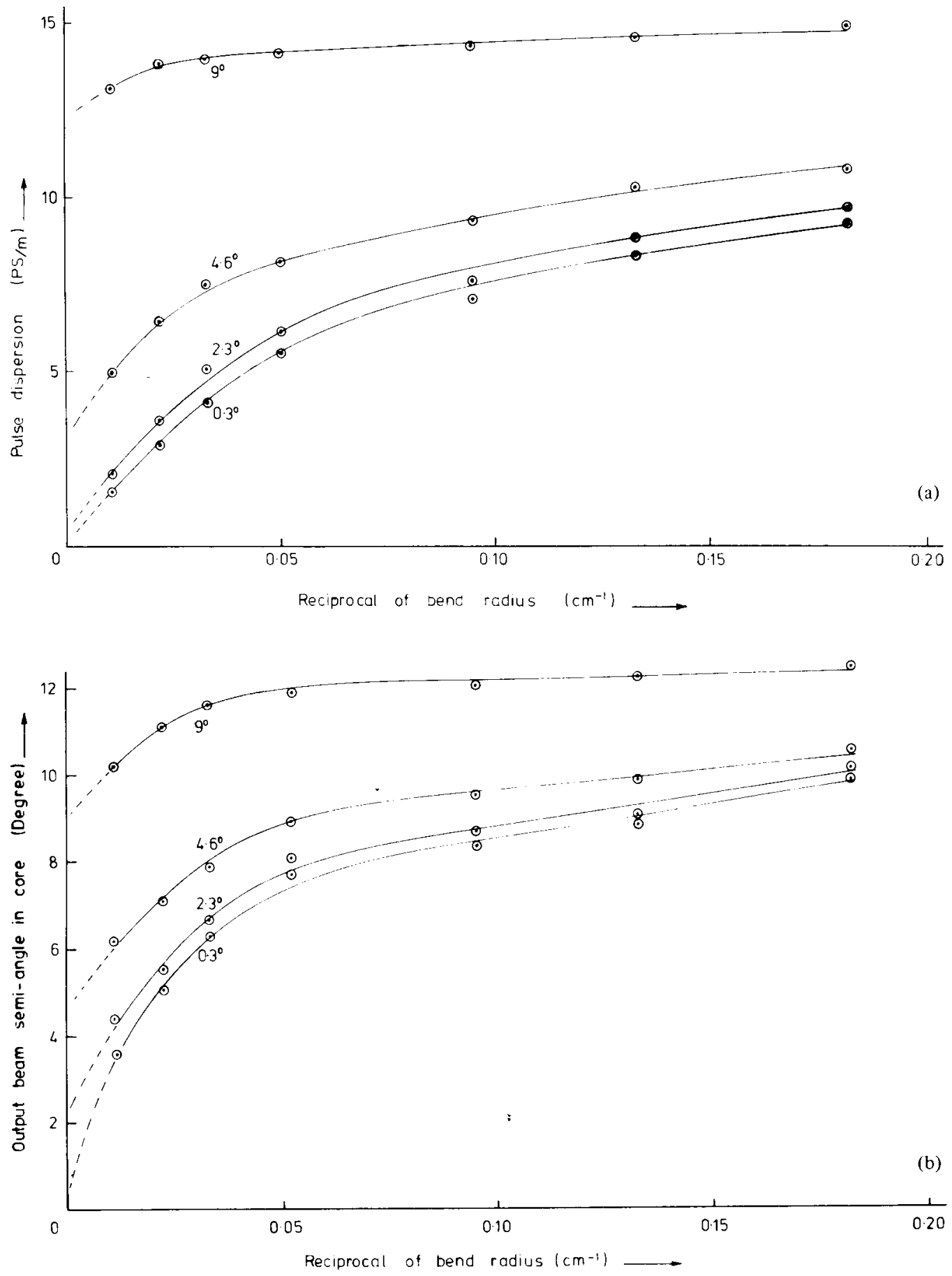

Fig. 1. (a) Pulse dispersion as a function of reciprocal bend radius for the input angular widths in the core shown on the curves. (b) Angular distribution in the core at the output end of the fibre as a function of reciprocal bend radius for various angular widths in the core at the input. 

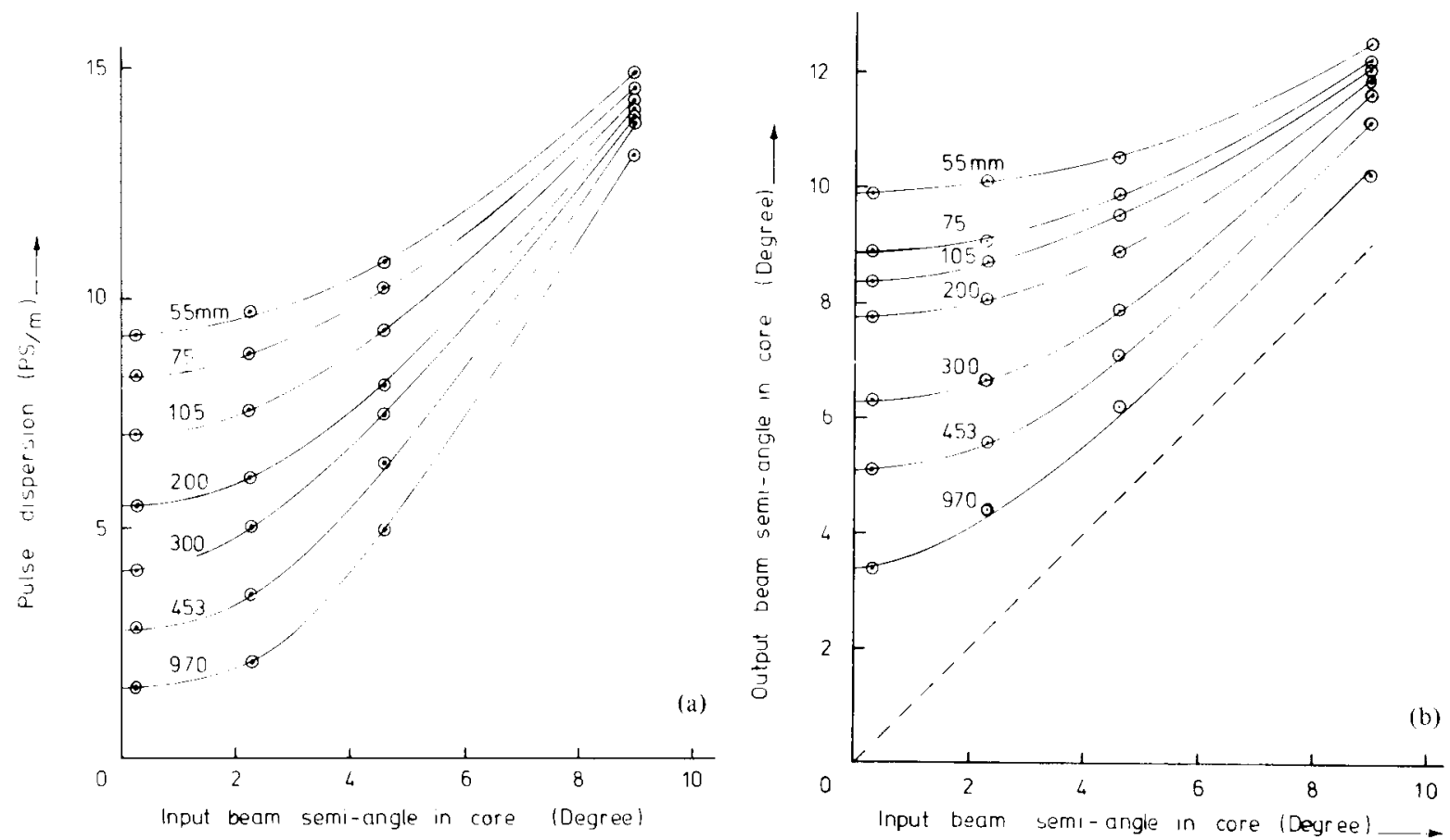

Fig. 2. (a) Pulse dispersion as a function of semi-angular width in the core at the input for various bend radii. (b) (urves for various bend radii showing increase in angular distribution of light in core from input to output for a $200 \mathrm{~m}$ length.

of the fibre but it is also interesting to examine the effect of the input beam semi-cone angle since this will indicate the various performances likely to be given by the different types of light source, such as single-mode lasers, multimode lasers and light-emitting diodes. Fig. 2a gives curves of dispersion as a function of input beam angular width for various bend radii and shows that the dispersion increases rapidly for beam semiangles greater than $3^{\circ}$. This is similar to the result we obtained with glass fibres. As expected the dispersion is less affected by bending radius at large beam solid angles. $A$ beam of $1^{\circ}$ semi-angular width in a straight fibre is likely to give a bandwidth approaching $1 \mathrm{GHz} /$ $\mathrm{km}$ whereas to achieve the still appreciable bandwidth of $100 \mathrm{MHz} / \mathrm{km}$ a beam semiangle of $6^{\circ}$ and a bend radius of $20 \mathrm{~cm}$ can be tolerated. Naturally the results quoted here are the lower limits since they were obtained with the entire length of fibre bent into the same curvature. In practice the main part of a practical fibre would be (relatively) straight and only a small portion would be curved, so that the bandwidth could be greater than the values quoted above.
The bandwidth could also depend on the position of bends in the fibre, as illustrated by measurements carried out with a $150 \mathrm{~m}$ length of which $100 \mathrm{~m}$ was wound on a drum of $5.5 \mathrm{~cm}$ radius and $50 \mathrm{~m}$ at $1 \mathrm{~m}$ radius. When pulses were introduced in to the end on the smaller drum the dispersion at the output was 1.7 nsec. On reversing the direction of propagation the dispersion was more than halved to $0.7 \mathrm{nsec}$.

Fig. 2b shows how the output beam semi-angle, converted to values corresponding to the angular distribution of light in the core, depends on the input beam width and again the curves show a strong similarity to those for dispersion in fig. 2a. The dashed line is the theoretical curve assuming a straight fibre and no mode conversion. As before the effect of bend radius is least, and the approach to the theoretical values is closest, at large input semi-angles. The angular light distribution at the output is no longer of gaussian shape.

Finally the effect of bend radius on relative propagation delay of the peak of the transmitted pulse as a function of the input angle of incidence for a beam of $0.3^{\circ}$ semi-angular width is shown in fig. 3 . This is of 


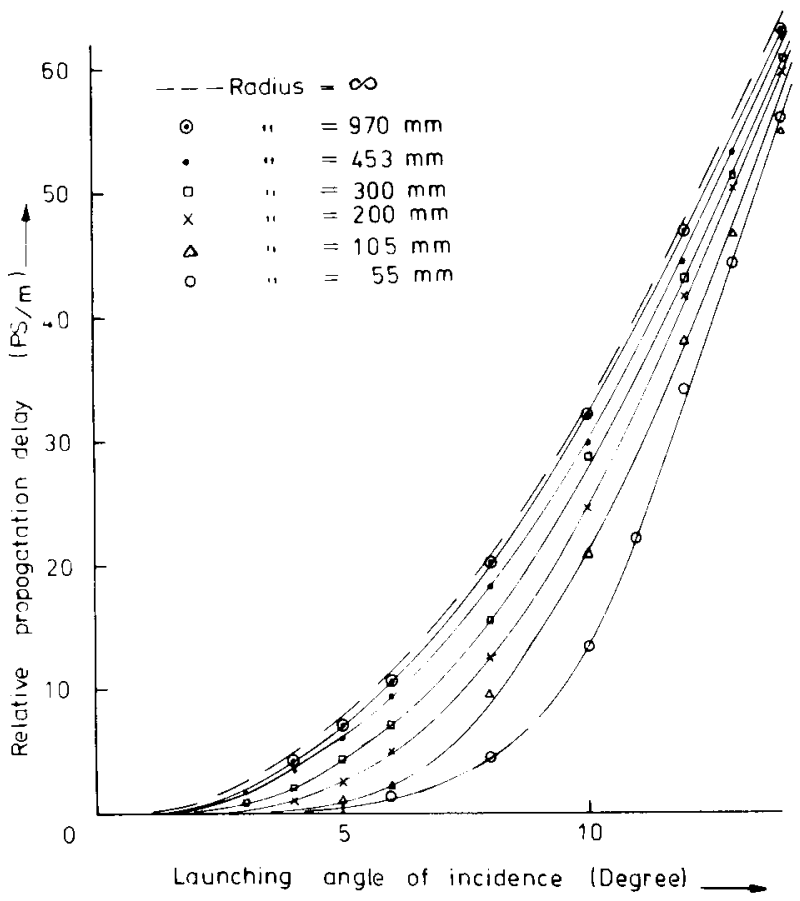

Fig. 3. Relative propagation delay as a function of angle of incidence for a $180 \mathrm{~m}$ length. The dashed curve is calculated from eq. (1) and corresponds to a straight fibre. The experimental points correspond to the following bend radii:

$---=\infty, \odot=970, \cdot=453, \square=300, x=200, \Delta=105$, $\mathrm{O}=55 \mathrm{~mm}$.

interest since the simple ray propagation model, which has been used to predict pulse dispersion, leads to an expression for the propagation delay of the form

$\Delta t=(n L / c)\left[\sec \left\{\sin ^{-1}(\sin \phi / n)\right\}-1\right]$,

where $n=$ refractive index of the core (1.551), $L=$ length of fibre $(180 \mathrm{~m})$ and $\phi=$ launching angle of incidence on the fibre. As may be seen there is good agreement with this equation at large bend radius but for more sharply curved fibres the propagation delay is decreased.

It follows therefore that for the lengths of fibres used in these experiments there is a progressive departure from the above equation with decreasing bend radius and a corresponding change in angular distribution of light from that in a straight fibre. Analysis of the equivalent two-dimensional model of a curved slab shows that this effect is not unexpected, for example the path length of a central ray decreases as the slab is ben $t$, leading to a reduced propagation time.

\section{Conclusions}

(1) The attenuation of fibres comprising hexachlorobuta-1,3,-diene in ME1 glass of $50 \mu \mathrm{m}$ core diameter has been reduced to $7 \mathrm{~dB} / \mathrm{km}$, measured over a length of $1 \mathrm{~km}$. (2) The measured pulse dispersion depends on the angular wid th as well as the angle of incidence of the input beam, i.e., on the number of modes launched. In a straight fibre this is probably the main dispersive mechanism. (3) Bending of the fibre causes mode conversion to occur. The dispersion, and the angular distribution of light in the fibre, both fall rapidly for bend radii greater than $20 \mathrm{~cm}$. (4) With a bend radius of $1 \mathrm{~m}$ a dispersion corresponding to a bandwidth of nearly $1 \mathrm{GHz}$ has been achieved. (5) No limitation on the bandwidth due to scattering or inhomogeneities either in the core or at the core/cladding interface has yet been observed although the maximum bandwidth in a straight fibre will presumably be limited by such fibre imperfections rather than dispersion of the bulk core material.

A significant feature of the above results is the fact that low loss and wide bandwidth is possible in a fibre having a high-loss cladding. For the fibre described here the estimated contribution of the cladding to the total loss is only $3 \mathrm{~dB} / \mathrm{km}$ [6] for the conditions of our loss measurements in which [2] the width of the transmitted incoherent beam was $\pm 6.9^{\circ}$. This result is consistent with the total loss value of $7 \mathrm{~dB} / \mathrm{km}$.

Although the dispersion measurements have been made at a wavelength of $0.633 \mu \mathrm{m}$ where the fibre attenuation is $50 \mathrm{~dB} / \mathrm{km}$ there seems to be no reason to suppose that the results will be markedly different at other wavelengths. Any mode filtering action caused by the core attenuation is neglible. For example the difference in at tenuations of an axial ray and a ray at $5^{\circ}$ to the axis is only $0.04 \mathrm{~dB}$ for the above core loss and thus any selective angular attenuation (i.e., mode filtering) will be comple tely dominated by the cladding. Again it was found experimentally that a change in bend radius from 5.5 to $30 \mathrm{~cm}$ greatly reduces the number of propagating modes and the dispersion falls from 9 to $4 \mathrm{psec} / \mathrm{m}$; at the same time the attenuation is reduced only from 10 to $8 \mathrm{~dB} / \mathrm{km}$ for the particular 
fibre measured. Thus the effect of mode filtering on the attenuation is small.

There is possibly a virtue, therefore, in having a high-loss cladding in that the mode-filtering action in selectively absorbing high-order modes can be used to maintain a wide bandwidth. The technique may, in some cases, be preferable to the alternative method of decreasing the numerical aperture of the fibre, as is sometimes done when a low-loss cladding is used, in order to reduce the number of modes capable of propagating. In the latter case bends, particularly near the input end of the fibre, and other causes of mode conversion result in loss by radiation in to the cladding once the acceptance angle of the fibre is filled. The angular distribution of light in the core then stays nearly constant for the remaining straight length of the fibre. On the other hand in a fibre such as ours, with a large numerical aperture and a high loss cladding then higher order modes which may be formed are selectively and progressively attenuated along the length of the fibre, resulting in an improved angular distribution of light and hence a decrease in dispersion. We are in the process of determining the magnitude of this effect in our fibres.

When cladded fibres were first suggested as a possible optical transmission medium it seemed likely that the bandwidth which could be achieved in practice, particularly in multimode fibres, would be limited by fibre imperfections. The results reported here show that liquid-core fibres, at least, behave like normal high-quality, overmoded transmission structures. A theoretical analysis which includes the effect of core and cladding loss, and a comparison with experiment, is being published elsewhere.

\section{Acknowledgement}

This work is supported by Science Research Council. One of the authors (HM) gratefully acknowledges partial support from the British Council.

\section{References}

[1] W.A. Gambling, D.N. Payne and H. Sunak, Electron. Letters 7 (1971) 549.

[2] D.N. Payne and W.A. Gambling, Electron. Letters 8 (1972) 374.

[3] R.D. Maurer, Paper presented at the 1st European ElectroOptics Conf. and Exhibition, Geneva (1972).

[4] W.A. Gambling, J.P. Dakin, D.N. Payne and H.R.D. Sunak, Electron. Letters 8 (1972) 260.

[5] D. Williams, private communication.

[6] R. Roberts, private communication. 